\title{
Design and Optimization of a Novel Compact Broadband Linearly/Circularly Polarized Wide-Slot Antenna for WLAN and WiMAX Applications
}

\author{
Ubaid ULLAH ${ }^{1}$, Slawomir KOZIEL $L^{1,2}$ \\ ${ }^{1}$ Engineering Optimization and Modeling Center of Reykjavik University, Reykjavik, Iceland \\ ${ }^{2}$ Faculty of Electronics, Telecommunications and Informatics, Gdansk University of Technology, 80-233 Gdansk, Poland \\ ubaidu@ru.is,koziel@ru.is
}

Submitted August 4, 2018 / Accepted January 16, 2019

\begin{abstract}
A novel topologically modified structure of a compact low profile wide-slot antenna for broadband applications is presented. The antenna comprises a modified E-shaped slot with unequal arm lengths in the ground plane, and a parasitic quasi-rectangular loop placed coplanar with the feedline. For exciting orthogonal modes with equal amplitude, a single-point feeding technique with an asymmetrical geometry of the coplanar waveguide is used to feed the antenna. A multi-stage EM-driven optimization is used to rigorously optimize all the parameters for best impedance bandwidth and axial ratio bandwidth. The total footprint of the antenna is only $0.71 \lambda_{\mathrm{g}}{ }^{2}$ and a $41 \%$ impedance bandwidth along with $33 \%$ axial ratio bandwidth has been achieved which covers several WLAN and WiMAX bands.
\end{abstract}

\section{Keywords}

Broadband antenna, wide-slot antenna, circular polarization, WLAN antenna, WiMAX antenna, EMdriven optimization

\section{Introduction}

Recent trends in wireless communication systems create the needs for compact size components, stable communication links, as well as high-speed data transmission [1-4]. As planar broadband circularly polarized (CP) antennas with compact footprints and low profiles are capable of fulfilling these requirements, therefore development of high-performance CP structures has become a hot research topic. Compact size and planar geometry make them a viable choice for integration with microwave integrated circuits, whereas CP characteristics are especially suitable in the context of mitigating multipath losses, dropping signal interference, polarization mismatch, as well as reducing Faradays rotation effects [5-7]. For high-speed wireless data transmission, simultaneous multi-channel communication and improved sensitivity between the transmitting and receiving antennas, a wide impedance bandwidth and axial ratio bandwidth (ARBW) of the antenna, are highly preferred.

Generally, CP polarization of the antenna can be attained with single point feeding but using this type of feeding with conventional geometry of the antenna can yield only a fraction of the impedance bandwidth. Similarly, if two-point feeding technique or addition active components are added to the circuit then it compromises the compactness of antenna and increases circuit complexity [8]. There are number of techniques that have been used for enhancing axial ratio bandwidth in particular [9], [10]. With the introduction of various topological modifications, an improvement in antenna performance (both in terms of electrical and field characteristics but also size reduction) can be obtained. However, these topological changes normally lead to increasing the structure complexity and hence a large number of geometrical parameters which have to be tuned. Unfortunately, most of the topologically modified planar antennas have a large number of parameters controlling the antenna performance figures such as impedance matching and axial ratio. Traditionally, the parameter sweeping techniques are used for optimizing all those adjustable parameters which is not an efficient approach both in terms of computational cost and also achieving a final finest design. To ensure optimum performance, rigorous numerical optimization of all antenna parameters is necessary, which, for the sake of reliability, has to be executed at the level of full-wave EM simulation models. This can be realized using conventional gradient-based algorithms [11], or, for improved computational efficiency, using surrogateassisted techniques [12].

In this paper, a novel geometry of a planar, compact size and broadband linearly/circularly polarized wide slot antenna is presented. The key features of wide impedance bandwidth and ARBW are achieved using four-stage topology evolution of the proposed antenna. A square wideslot antenna with a compact geometry of $25 \times 25 \mathrm{~mm}^{2}$ is used as a baseline design which is well-known for its wide impedance bandwidth and ARBW performance [13]. Sys- 
tematic topological modification is introduced at the periphery of the wide-slot etched in the ground plane for exciting additional $\mathrm{CP}$ modes and broadening the ARBW. The major performance figures, i.e. impedance bandwidth and ARBW, are further improved through rigorous EMdriven optimization of relevant geometry parameters. For a computationally efficient optimization, the adjustable variables are subdivided based on a thorough sensitivity analysis. In the first stage of optimization, the most sensitive variables are optimized followed by repeating the same optimization routine for the whole set of variables. $\mathrm{Nu}-$ merical results are validated experimentally with a close agreement between simulation and measurement results. Finally, a comprehensive benchmarking is carried out indicating advantages of the proposed antenna over state-ofthe-art designs reported in the literature.

\section{Antenna Configurations and Design Steps}

The proposed antenna is designed on a laminated Rogers substrate RO4003C that has permittivity of 3.38, tangent loss 0.0027 , and thickness of $0.813 \mathrm{~mm}$. In step one, a wide square slot antenna was designed which is fed by an asymmetric coplanar waveguide (CPW) as shown in Fig. 1(a). The length $L_{\mathrm{m}}$ of the microstrip-line monopole, extending from the CPW, was kept to approximately $\lambda / 4$ at the center operating frequency (with respect to the impedance bandwidth) of the antenna. In the second step, the geometry of the square slot was modified, and a perturbation was introduced to enable generation of the orthogonal current distribution on the contour of the slot. The final design of the slot after the perturbation is of an E-shape with unequal arm lengths, which primarily contributes to the excitation of the orthogonal modes. In the conventional slot, the current only flows in one direction, along the $y$ plane, as shown in Fig. 1(b). By introducing a perturbation of unequal length extends, the current path and the $y$-plane as well as asymmetric current components in the $x$-plane are also generated at the slot contour as illustrated in Fig. 1(c). The magnitude of the asymmetric current in the $x y$-plane is almost of equal magnitude but shifted $90^{\circ}$ in phase, which allows for generating additional CP modes. To achieve sufficient ARBW, a quasi-rectangular loop of a length equivalent to $\lambda / 4$ (at a lower cutoff frequency), is placed coplanar to the monopole. This parasitic component directs the current in the horizontal and vertical plane and, therefore, induces $\mathrm{CP}$.

The final design step consists of adding another parasitic metallic strip within the wide slot in the ground plane, and a small impedance matching stub to the feed-line monopole. The main contribution of this step is improving the impedance matching by lengthening the current path which, as shown in Fig. 2, enhances both the impedance bandwidth and ARBW of the proposed antenna. The length of the parasitic strips and slots used in the proposed design are indicated with $L_{n}$ and $L_{\mathrm{c} n}$ while their corresponding widths

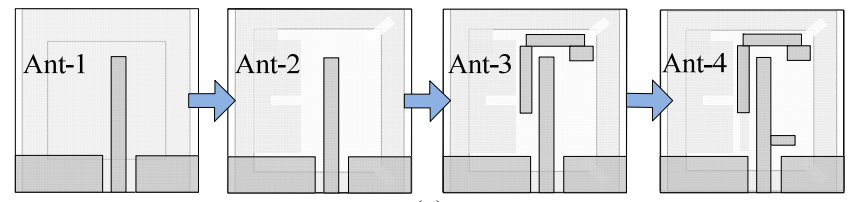

(a)

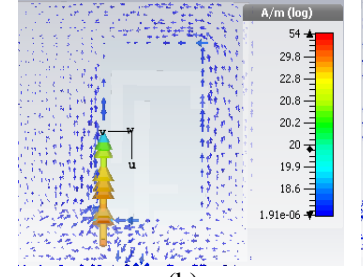

(b)

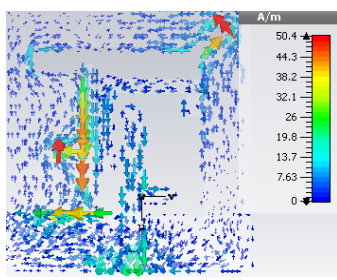

(c)

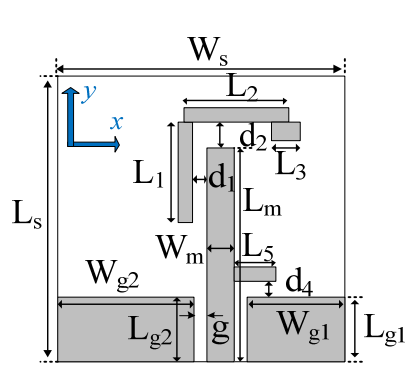

(d)

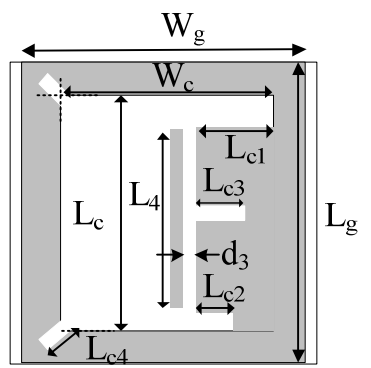

(e)
Fig. 1. Antenna evaluation stages and final parametrized geometry: (a) Evolution stages of the proposed antenna. (b) Surface current distribution on conventional slot. (c) Surface current distribution on perturbed slot. (d) Parameterized front view. (e) Parameterized back view.

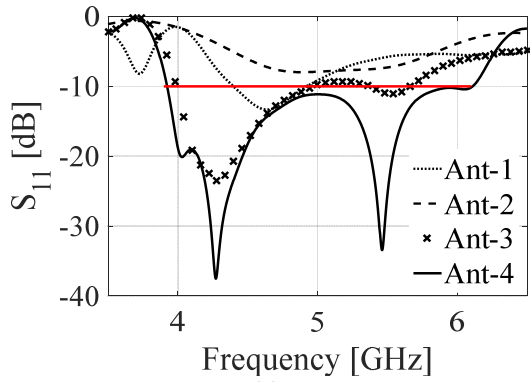

(a)

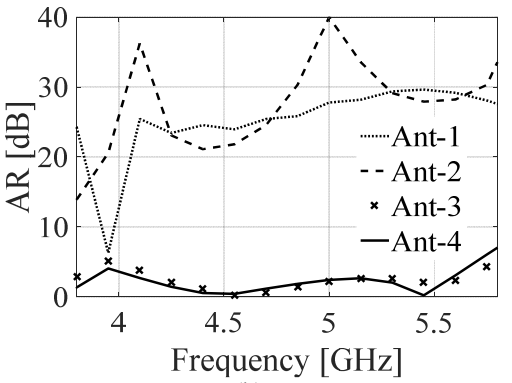

(b)

Fig. 2. Simulated characteristics of the optimized design of antenna of Fig. 1. (a) Reflection responses at four evolution stages. (b) Axial ratio characteristics at four evolution stages.

are represented as $W_{n}$ and $W_{\mathrm{c} n}$, where, $n=1,2,3 \ldots$ The four evolution stages and the final parameterized design are depicted in Fig. 1. The geometrical size of the proposed 
antenna is $25 \mathrm{~mm} \times 25 \mathrm{~mm}$ (the footprint of only $625 \mathrm{~mm}^{2}$ ), see also Tab. 1 .

\subsection{Design Problem and Optimization Algorithm}

Appropriate optimization of antenna geometry parameters is critical for achieving the best possible performance. Here, a multi-stage process is utilized which includes the following steps. For the purpose of description, the antenna impedance bandwidth will be denoted as $B W_{S}(\boldsymbol{x})$ (i.e., the continuous frequency range for which $\left.\left|S_{11}\right| \leq-10 \mathrm{~dB}\right)$, whereas $S(\boldsymbol{x})$ will denote the maximum reflection level within the proposed antenna frequency range. Also, we use the symbol $A R(\boldsymbol{x})$ to denote the maximum value of axial ratio (AR) within the same frequency range. An explicit dependence on adjustable parameter vector $\boldsymbol{x}$ is indicated to facilitate further description.

The design specifications are as follows:

- Ensure that $S(\boldsymbol{x}) \leq-10 \mathrm{~dB}$;

- Maximize $B W_{S}(\boldsymbol{x})$ around $5 \mathrm{GHz}$;

- Minimize $A R(\boldsymbol{x})$

Generally, straightforward optimization of all parameters with respect to all specifications fails due to several issues which include: (i) high cost of EM simulation of the antenna structure, (ii) a large number of geometrical parameters, and (iii) the necessity of simultaneous handling of several performance figures. To alleviate these problems, a multi-stage procedure is utilized as described below. The generic optimization task is formulated as follows:

$$
\boldsymbol{x}^{*}=\arg \min _{\boldsymbol{x}} U\left(B W_{\mathrm{S}}(\boldsymbol{x}), S(\boldsymbol{x}), A R(\boldsymbol{x})\right)
$$

where $U$ is the objective function. We consider two separate sub-problems. The antenna impedance bandwidth optimization is the first step for which the objective function is defined as:

$$
U_{\mathrm{S}}\left(B W_{\mathrm{S}}(\boldsymbol{x}), S(\boldsymbol{x}), A R(\boldsymbol{x})\right)=-B W_{\mathrm{S}}(\boldsymbol{x})+\beta_{\mathrm{S}} c_{\mathrm{S}}(S(\boldsymbol{x}))^{2}
$$

in which $c_{\mathrm{S}}(S(\boldsymbol{x}))=\max \{(S(\boldsymbol{x})+10.0\} / 10$ is a penalty function that "measures" a relative violation of the condition $S(\boldsymbol{x}) \leq-10 \mathrm{~dB}$, and $\beta_{\mathrm{S}}$ is a penalty factor.

The purpose of solving (1) with the objective function (2) is to increase the antenna bandwidth and to ensure sufficient impedance matching within the antenna operational bandwidth.

The objective function for the second sub-problem where the goal is to minimize the axial ratio bandwidth below $3 \mathrm{~dB}$, is defined as:

$$
U_{\mathrm{AR}}\left(B W_{\mathrm{S}}(\boldsymbol{x}), S(\boldsymbol{x}), A R(\boldsymbol{x})\right)=A R(\boldsymbol{x})+\beta_{\mathrm{S}} c_{\mathrm{S}}\left(S^{\prime}(\boldsymbol{x})\right)^{2} .
$$

The symbol $S^{\prime}(\boldsymbol{x})$ denotes the maximum reflection level within the bandwidth obtained upon solving (1) with the objective function (2).
Furthermore, in order to facilitate the optimization process, sensitivity analysis was performed to identify the parameters with respect to which both design goals i.e. $\left|S_{11}\right|$ and $A R$ are the most sensitive. The twelve selected variables

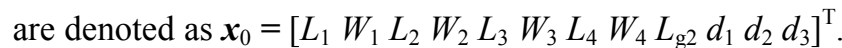
The remaining variables are included in the second design stage.

The optimization process was carried out with the following steps:

1. Solve the problem (1) with the objective function (2) for a reduced parameter set $\boldsymbol{x}_{0}$ :

$$
\boldsymbol{x}_{0}^{*}=\arg \min _{\boldsymbol{x}} U_{\mathrm{S}}\left(B W_{\mathrm{S}}\left(\boldsymbol{x}_{0}\right), S\left(\boldsymbol{x}_{0}\right), A R\left(\boldsymbol{x}_{0}\right)\right) .
$$

2. Starting from $\boldsymbol{x}_{0}{ }^{*}$, solve the problem (1) with the objective function (3) for a reduced parameter set:

$$
\boldsymbol{x}_{0}^{* *}=\arg \min _{\boldsymbol{x}} U_{\mathrm{AR}}\left(B W_{\mathrm{S}}\left(\boldsymbol{x}_{0}\right), S\left(\boldsymbol{x}_{0}\right), A R\left(\boldsymbol{x}_{0}\right)\right) .
$$

3. Solve the problem (1) with the objective function (2) for the entire parameter set (the components of $\boldsymbol{x}_{0}{ }^{* *}$ are used as initial values):

$$
\boldsymbol{x}^{*}=\arg \min _{\boldsymbol{x}} U_{\mathrm{S}}\left(B W_{\mathrm{S}}(\boldsymbol{x}), S(\boldsymbol{x}), A R(\boldsymbol{x})\right) .
$$

4. Identify the final design by solving (1) with the objective function (3) for the entire parameter set, starting from $\boldsymbol{x}^{*}$ :

$$
\boldsymbol{x}^{* *}=\arg \min _{\boldsymbol{x}} U_{\mathrm{AR}}\left(B W_{\mathrm{S}}(\boldsymbol{x}), S(\boldsymbol{x}), A R(\boldsymbol{x})\right) .
$$

All sub-problems are solved using a trust-region gradient search [14] with the antenna response gradients estimated using finite differentiation. Sequential optimization (first for impedance bandwidth enhancement, then for axial ratio improvement) allows for efficient handling of all performance figures. In particular, ensuring sufficient matching provides a feasible starting point for AR optimization. The results of three stage optimization are depicted in term of impedance matching and AR in Fig. 3 and Fig. 4 respectively. The initial design values are obtained based on classic antenna theory. As can be seen from reflection

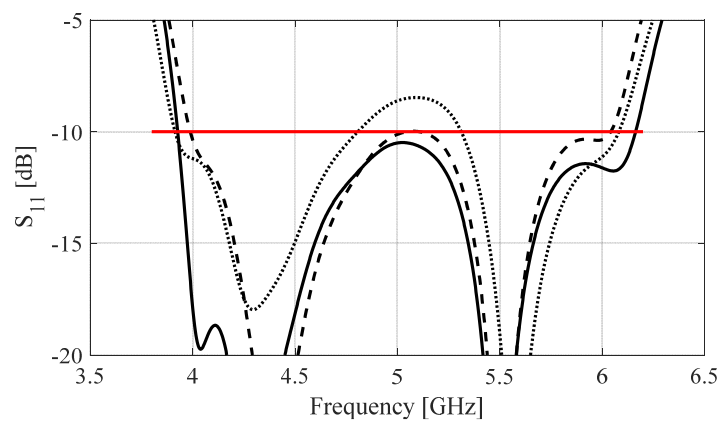

Fig. 3. Reflection response of the antenna of Fig. 1 at various stages of the design process: initial design $(\cdots)$, optimization with reduced parameter set $\boldsymbol{x}_{0}{ }^{* *}(--)$, final design $\boldsymbol{x}^{* *}(-)$. Bandwidth of interest and $-10 \mathrm{~dB}$ level marked with vertical and horizontal lines, respectively. 


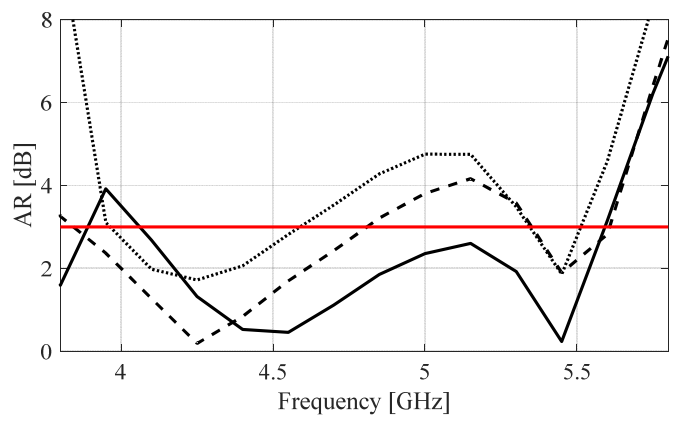

Fig. 4. Axial ratio of the antenna of Fig. 1 at various stages of the design process: initial design ( $\cdots)$, optimization with reduced parameter set $\boldsymbol{x}_{0}{ }^{* *}(--)$, final design $\boldsymbol{x}^{* *}$ (一). Bandwidth of interest and $3 \mathrm{~dB}$ level marked with vertical and horizontal lines, respectively.

\begin{tabular}{|c|c|c|c|c|c|c|}
\hline Parameter & $L_{\mathrm{s}}$ & $W_{\mathrm{s}}$ & $L_{\mathrm{g}}$ & $W_{\mathrm{g}}$ & $L_{\mathrm{g} 1}$ & $W_{\mathrm{g} 1}$ \\
\hline Value (mm) & 25 & 25 & 25 & 23 & 5 & 10.12 \\
\hline Parameter & $L_{\mathrm{g} 2}$ & $W_{\mathrm{g} 2}$ & $L_{\mathrm{m}}$ & $W_{\mathrm{m}}$ & $L_{1}$ & $W_{1}$ \\
\hline Value (mm) & 5.11 & 12.12 & 15.5 & 1.55 & 7.55 & 1.3 \\
\hline Parameter & $L_{2}$ & $W_{2}$ & $L_{3}$ & $W_{3}$ & $L_{4}$ & $W_{4}$ \\
\hline Value (mm) & 8.7 & 1.7 & 2.18 & 1.95 & 3.12 & 0.94 \\
\hline Parameter & $L_{\mathrm{c}}$ & $W_{\mathrm{c}}$ & $L_{\mathrm{c} 1}$ & $W_{\mathrm{c} 1}$ & $L_{\mathrm{c} 2}$ & $W_{\mathrm{c} 2}$ \\
\hline Value (mm) & 17.96 & 16.4 & 5.13 & 2.63 & 2.87 & 1.69 \\
\hline Parameter & $L_{\mathrm{c} 3}$ & $W_{\mathrm{c} 3}$ & $L_{\mathrm{c} 4}$ & $W_{\mathrm{c} 4}$ & $g$ & $d_{1}$ \\
\hline Value (mm) & 2.37 & 0.91 & 3.12 & 1.37 & 0.6075 & 1.656 \\
\hline Parameter & $d_{2}$ & $d_{3}$ & $d_{4}$ & $L_{5}$ & $W_{5}$ & \\
\hline Value (mm) & 1.03 & 0.57 & 1.56 & 3.12 & 0.94 & \\
\hline
\end{tabular}

Tab. 1. Parameter values of the proposed CP antenna.

coefficient response of the antenna, for the initial design the impedance matching is way above $-10 \mathrm{~dB}$ reference line. After the first stage optimization of most sensitive variables, both impedance matching and axial ratio are improved and reach near the reference line at $-10 \mathrm{~dB}$ and $3 \mathrm{~dB}$ respectively. After performing the final optimization for the whole set of variables the responses are further improved and the primary goal of well-matched antenna is achieved. It is important to mention here that a compact physical size of the antenna is maintained to $25 \times 25 \mathrm{~mm}^{2}$. It is very challenging to achieve a wide impedance bandwidth and axial ratio bandwidth with the traditionally used optimization techniques.

\section{Experimental Verification and Benchmarking}

The final design of the proposed antenna has been fabricated and measured. Figure 5 shows the photograph of the antenna prototype and the experimental setup for characterizing the proposed antenna. Similarly, a comparison of simulated and measured reflection response, axial ratio, and realized gain (both in a broadside direction) is illustrated

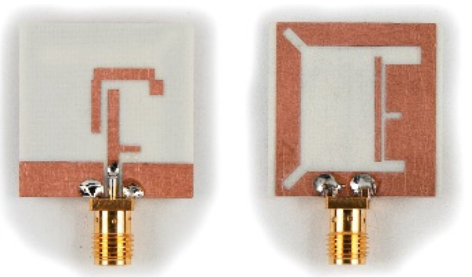

(a)

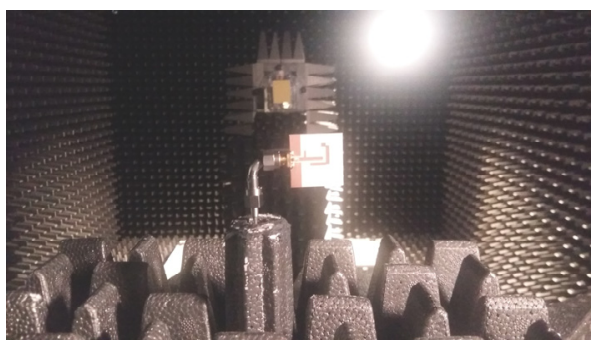

(b)

Fig. 5. Experimental validation of the proposed CP antenna: (a) Photographs of the antenna prototype (front - left, back - right). (b) Experimental setup.

in Fig. 6. An impedance bandwidth of $44 \%(3.9 \mathrm{GHz}$ to $6.2 \mathrm{GHz}$ ) is achieved with a $34 \%$ ARBW while a compact geometry of the antenna is maintained. Furthermore, the antenna exhibits a stable gain response throughout the operating band and the average realized gain of the antenna is $3.62 \mathrm{~dB}$. The impedance bandwidth and axial ratio bandwidth response shows that the antenna can be used for several applications around $5 \mathrm{GHz}$ frequency which include WLAN and WiMAX band.

Figure 7 shows the LHCP and RHCP radiation patterns of the antenna in $x z$-plane. Similarly, Figure 8 depicts the simulated and measured $x y$-plane. A bidirectional radiation pattern with good agreement between simulation and measurement is observed. Slight discrepancies originate from fabrication/assembly inaccuracies and mechanical instabilities.

\section{Benchmarking}

The proposed antenna has been compared in terms of ARBW, impedance bandwidth, and footprint to several state-of-the-art $\mathrm{CP}$ antennas recently reported in the literature. The structures operating within similar frequency ranges have been selected. For the sake of fair comparison, the antenna sizes were expressed in terms of the guided wavelength $\left(\lambda_{\mathrm{g}}\right)$. Table 2 indicates that the proposed antenna exhibits better performance with respect to all major performance figures, including impedance bandwidth, ARBW, and the size.

\section{Conclusion}

The article proposes a novel structure of a compact low profile wide-slot CP antenna. A multi-stage optimization routine is developed and implemented for rigorously 


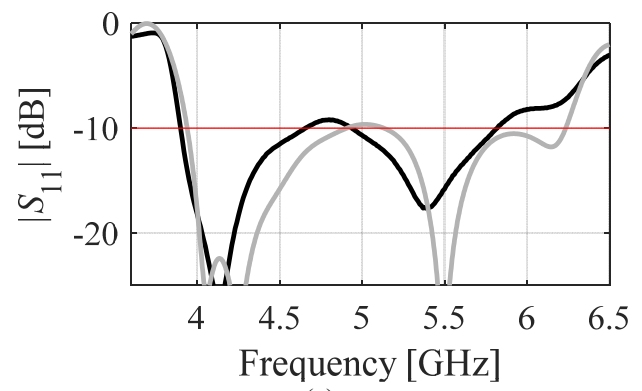

(a)

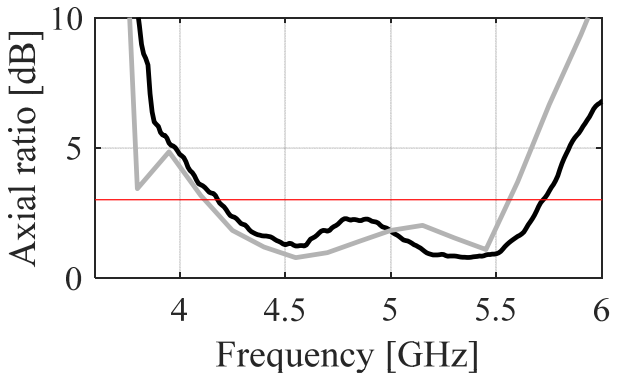

(b)

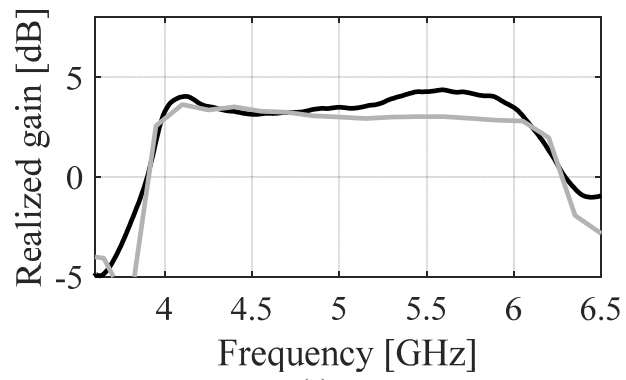

(c)

Fig. 6. Experimental validation of the proposed CP antenna: (a) Simulated (gray) and measured (black) reflection responses. (b) Simulated (gray) and measured (black) axial ratio characteristics. (c) Simulated (gray) and measured (black) realized gain characteristics.

\begin{tabular}{|c|c|c|c|c|c|}
\hline \multirow{2}{*}{ Ref. } & \multirow{2}{*}{$\begin{array}{c}\% \\
\mathbf{A R}^{\$}\end{array}$} & \multirow{2}{*}{$\begin{array}{c}\% \\
\text { IBW }^{*}\end{array}$} & \multicolumn{2}{|c|}{ Antenna footprint } & \multirow{2}{*}{ Year } \\
\hline & & & $\mathrm{mm}^{2}$ & $\lambda_{g}{ }^{2 \#}$ & \\
\hline [15] & 27 & 111 & 2500 & 1.58 & 2013 \\
\hline$[16]$ & 15.4 & ---- & 900 & 0.95 & 2010 \\
\hline [17] & 26.9 & 27 & 2500 & 1.58 & 2017 \\
\hline [18] & 14 & 27 & 400 & 0.63 & 2015 \\
\hline [19] & 30 & 51 & 19158 & 1.44 & 2013 \\
\hline [20] & 22 & 37 & 2500 & 2.00 & 2015 \\
\hline [21] & 22.2 & 28.6 & 2025 & 54.56 & 2017 \\
\hline $\begin{array}{l}\text { This } \\
\text { work }\end{array}$ & 33 & 41 & 625 & 0.71 & 2018 \\
\hline
\end{tabular}

Tab. 2. Comparison with state-of-the-art CP antennas. $\$ \%$ AR stands for fractional axial ratio bandwidth, * \% IBW stands for fractional impedance bandwidth, \# $\lambda_{g}{ }^{2}$ denotes the antenna footprint in terms of the guided wavelength.

optimizing all adjustable parameters. The final optimized design is then prototyped and characterized in anechoic chamber. The antenna features 33-percent axial ratio bandwidth and 41-percent impedance bandwidth as well as a small size of only $25 \mathrm{~mm} \times 25 \mathrm{~mm}$. The operational range covers several WLAN and WiMAX bands. Comprehensive benchmarking indicates that the proposed antenna

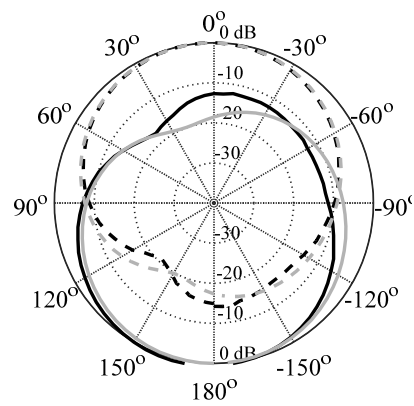

(a)

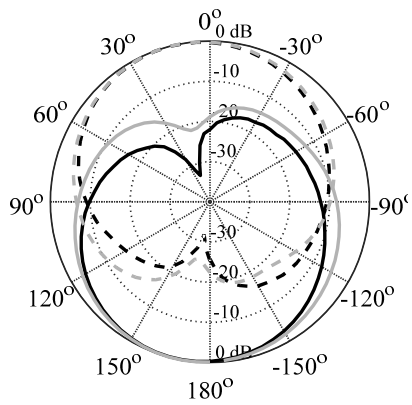

(c)

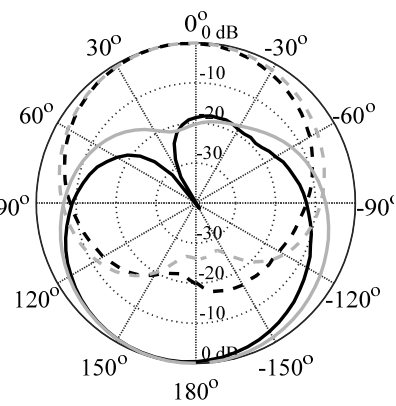

(b)

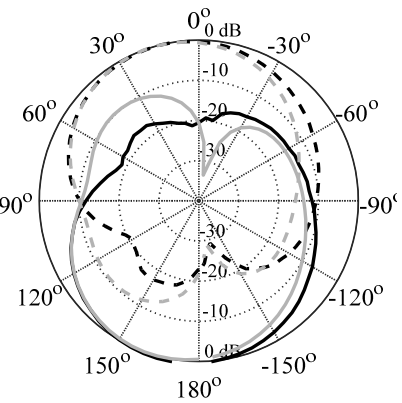

(d)
Fig. 7. Simulated (grey) and measured (black) radiation patterns obtained for the proposed antenna in the $x z$ plane. RHCP and LHCP patterns are shown using solid and dashed lines, respectively (a) $4.2 \mathrm{GHz}$, (b) $4.9 \mathrm{GHz}$, (c) $5.2 \mathrm{GHz}$, (d) $5.5 \mathrm{GHz}$.

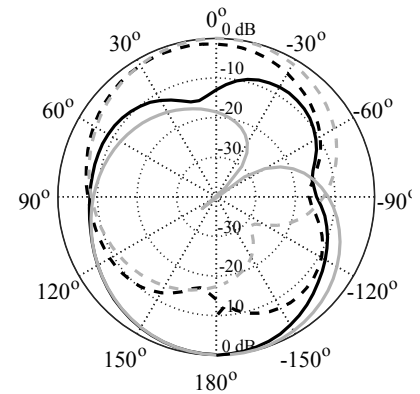

(a)

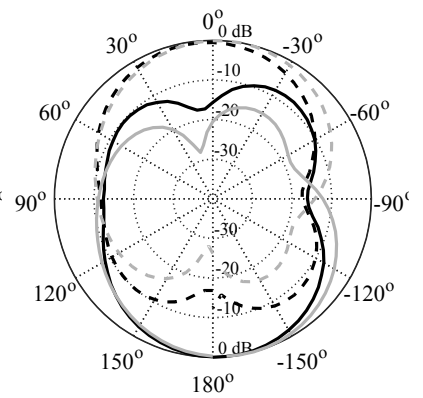

(b)

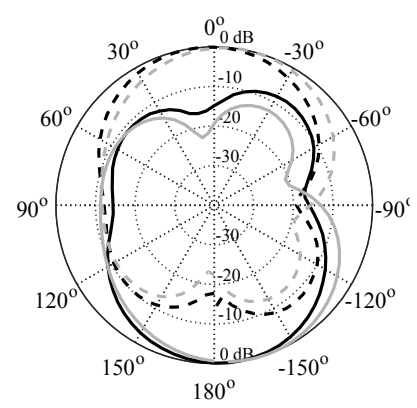

(c)

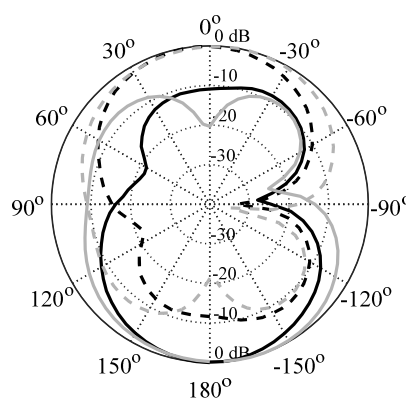

(d)
Fig. 8. Simulated (grey) and measured (black) radiation patterns obtained for the proposed antenna in the $x y$ plane. RHCP and LHCP patterns are shown using solid and dashed lines, respectively (a) $4.2 \mathrm{GHz}$, (b) $4.9 \mathrm{GHz}$, (c) $5.2 \mathrm{GHz}$, (d) $5.5 \mathrm{GHz}$.

exhibits competitive performance compared to state-of-theart structures from the literature. 


\section{Acknowledgment}

The authors would like to thank Dassault Systemes, France, for making CST Microwave Studio available. This work was supported in part by the Icelandic Centre for Research (RANNIS) Grant 174114051.

\section{References}

[1] MAHMOUD, K. R., MONTASER, A. M. Design of dual-band circularly polarised array antenna package for $5 \mathrm{G}$ mobile terminals with beam-steering capabilities. IET Microwave Antennas and Propagation, 2018, vol. 12, no. 1, p. 29-39. DOI: 10.1049/iet-map.2017.0412

[2] YANG, Z. J., XIAO, S. Q., ZHU, L., et al. A circularly polarized implantable antenna for 2.4-GHz ISM band biomedical applications. IEEE Antennas and Wireless Propagation Letters, 2017, vol. 16, no. 1, p. 2554-2557. DOI: 10.1109/LAWP.2017.2732460

[3] ULlah, U., AIN, M. F., OTHMAN, A., et al. A novel multipermittivity cylindrical dielectric resonator antenna for wideband applications. Radioengineering, 2014, vol. 23, no. 4, p. 1071-1076. ISSN: 1210-2512

[4] BANERJEE, S., RANA, B., PARUI, S. K. Gain augmentation of a HMSIW based equilateral triangular antenna using CRSF FSS superstrate. Radioengineering, 2018, vol. 27, no. 1, p. 47-53. DOI: $10.13164 /$ re.2018.0047

[5] VASINA, P., LACIK, J. Circularly polarized rectangular ring-slot antenna with chamfered corners for off-body communication at $5.8 \mathrm{GHz}$ ISM band. Radioengineering, 2017, vol. 26, no. 1, p. 85-90. DOI: 10.13164/re.2017.0085

[6] KUMAR, S., VISHWAKARMA, R. K., KUMAR, R. J., et al. Slotted circularly polarized microstrip antenna for RFID application. Radioengineering, 2017, vol. 26, no. 4, p. 1025-1032. DOI: 10.13164/re.2017.1025

[7] MADDIO, S. Parasitic-enhanced circularly polarised sequential antenna array for dedicated short-range communication applications at 5.8 GHz. Electronic Letters, 2017, vol. 53, no. 13, p. 824-826. DOI: $10.1049 / \mathrm{el} .2017 .0679$

[8] ULLAH, U., KOZIEL, S. Design and optimization of a novel miniaturized low-profile circularly polarized wide-slot antenna. Journal of Electromagnetic Waves and Applications, 2018, vol. 32, no. 16, p. 2099-2109. DOI: 10.1080/09205071.2018.1496039

[9] PAZOKI, R., KIAEE, A., NASERI, P., et al. Circularly polarized monopole L-shaped slot antenna with enhanced axial-ratio bandwidth. IEEE Antenna and Wireless Propagation Letters, 2016, vol. 15, p. 1073-1076. DOI: 10.1109/LAWP.2015.2492918

[10] LI, Y., ZHANG, Z., FENG, Z. A sequential-phase feed using a circularly polarized shorted loop structure. IEEE Transactions on Antennas and Propagation, 2013, vol. 61, no. 3, p. 1443-1447. DOI: 10.1109/TAP.2012.2227103

[11] KOZIEL, S., OGURTSOV, S. Model management for costefficient surrogate-based optimisation of antennas using variablefidelity electromagnetic simulations. IET Microwaves, Antennas and Propagation, 2012 vol. 6, no. 15, p. 1643-1650. DOI: 10.1049/iet-map.2012.0222

[12] BEKASIEWICZ, A., KOZIEL, S., Structure and computationallyefficient simulation-driven design of compact UWB monopole antenna. IEEE Antenna and Wireless Propagation Letters, 2015, vol. 14, no. 1, p. 1282-1285. DOI: 10.1109/LAWP.2015.2402282

[13] NOSRATI, M., TAVASSOLIAN, N. Miniaturized circularly polarized square slot antenna with enhanced axial-ratio bandwidth using an antipodal Y-strip. IEEE Antennas Wireless Propagation
Letters, 2017, vol. 16, no. 1, p. 817-820. DOI: 10.1109/LAWP.2016.2605099

[14] KOZIEL, S., BANDLER, J. W., CHENG, Q. S. Robust trustregion space-mapping algorithms for microwave design optimization. IEEE Transactions on Microwave Theory and Techniques, 2010, vol. 58, no. 8, p. 2166-2174. DOI: 10.1109/TMTT.2010.2052666

[15] JAN, J. Y., PAN, C. Y., CHIU, K. Y., et al. Broadband CPW-fed circularly-polarized slot antenna with an open slot. IEEE Transactions on Antennas and Propagation, 2013, vol. 61, no. 3, p. 1418-1422. DOI: 10.1109/TAP.2012.2231926

[16] BEIGMOHAMMADI, G., GHOBADI, C., NOURINIA, J., et al. Small square slot antenna with circular polarisation characteristics for WLAN/WiMAX applications. Electronic Letters, 2010, vol. 46, no. 10, p. 672-673. DOI: 10.1049/el.2010.0623

[17] CHOWDHURY, R., KUMAR, R., CHAUDHARY, R. K. A coaxial probe fed wideband circularly polarized antenna using unequal and adjacent-slided rectangular dielectric resonators for WLAN applications. International Journal of RF and Microwave Computer Aided Engineering, 2017, p. 1-9. DOI: $10.1002 /$ mmce. 21210

[18] OJAROUDI, Y., OJAROUDI, N., GHADIMI, N. Circularly polarized microstrip slot antenna with a pair of spur-shaped slits for WLAN applications, Microwave and Optical. Technology Letters, 2015, vol. 57, p. 756-759. DOI: 10.1002/mop.28946

[19] TRIVESH, K., HARISH, A. R. Broadband circularly polarized printed slot-monopole antenna. IEEE Antennas and Wireless Propagation Letters, 2013, vol. 12, no. 1, p. 1531-1534. DOI: 10.1109/LAWP.2013.2291436

[20] FAKHTE, S., ORAIZI, H., KARIMIAN, R., et al. A new wideband circularly polarized stair-shaped dielectric resonator antenna. IEEE Transactions on Antennas and Propagation, 2015, vol. 63 , no. 4 , p. $1828-1832$. DOI: 10.1109/TAP.2015.2392131

[21] DU, M., XU, J., DONG, Y., et al. Low-cost and high-gain SIW circularly polarized circular-horn-loaded antenna for broadband millimeter-wave applications. Radioengineering, 2017, vol. 26, no. 3, p. 728-734. DOI: 10.13164/re.2017.0728

\section{About the Authors ...}

Ubaid ULLAH received the M.Sc. and Ph.D. degrees in Electrical and Electronic Engineering from Universiti Sains Malaysia, Malaysia in 2013 and 2017, respectively. His research interest includes circular polarization, wideband antennas, and dielectric resonator antenna, CAD modeling of antenna structures, simulation-driven design, and surrogatebased optimization. He is currently working with the Engineering Optimization and Modelling Center, School of Science and Engineering, Reykjavik University, Iceland.

Slawomir KOZIEL received the M.Sc. and Ph.D. degrees in Electronic Engineering from Gdansk University of Technology, Poland, in 1995 and 2000, respectively. He received the M.Sc. degrees in Theoretical Physics and in Mathematics, in 2000 and 2002, respectively, as well as the Ph.D. in Mathematics in 2003, from the University of Gdansk, Poland. He is currently a Professor. His research interests include CAD and modeling of microwave and antenna structures, simulation-driven design, surrogate-based optimization, space mapping, circuit theory, analog signal processing, evolutionary computation and numerical analysis. 